\title{
Nanoscale Rotational Dynamics of Four Independent Rotators Confined in Crowded Crystalline Layers
}

Antonio Rodríguez-Fortea, ${ }^{\text {a }}$ Enric Canadell, ${ }^{* b}$ Pawel Wzietek, ${ }^{* \mathrm{c}}$ Cyprien Lemouchi, ${ }^{\mathrm{d}}$ Magali Allain, ${ }^{\mathrm{d}}$ Leokadiya Zorina, ${ }^{\mathrm{e}}$ and Patrick Batail*d

We report a study where Car-Parrinello molecular dynamics simulations and variable-temperature (30-300 $\mathrm{K}){ }^{1} \mathrm{H}$ spin-lattice relaxation time experiments nicely complement each other to characterize the dynamics within a set of four crystalline 1,4-Diethynylbicyclo[2.2.2]octane (BCO) rotors assembled in the metal-organic rotor, $\left\{\mathrm{Li}_{4}^{+}\left({ }^{-} \mathrm{CO}_{2}-\mathrm{Ph}-\mathrm{BCO}-\mathrm{py}\right)_{4}\left(\mathrm{H}_{2} \mathrm{O}\right)_{8}\right\} \cdot 2 \mathrm{DMF}$. The remarkable finding of this work is that, despites the individual rotational barriers of four rotors being undiscernible, superimposed in a broad relaxation process, we were able to unravel a strongly interrelated series of rotational motions involving disrotatory and conrotatory motions in pairs as well as rotational steps of single rotators, all three processes with similar, sizeable rotational barriers of $6 \mathrm{kcal} \mathrm{mol}^{-1}$. It is noteworthy that DFT molecular dynamics simulations and variable-temperature (30-300 K) proton spin-lattice relaxation time experiments deliver the same high value for the rotational barriers stressing the potential of the combined use of the two techniques in understanding rotational motion at the nanoscale.

\section{Introduction}

Steps towards understanding the mechanism of motion at the nanoscale of nanomotors or Brownian oscillators, be they soft matter objects, ${ }^{1,2}$ molecular machines, ${ }^{3-12}$ or crystalline rotors, $^{7-12}$ need to be taken in order to design ways to get control over these complex systems ${ }^{1,2}$ by driving them out of equilibrium in a deliberate manner. Sizeable molecular units, much bulkier than a methyl group, can rotate in the solid state as fast as in the gas phase, as exemplified by the solid state chemistry and physics of rotors with a 1,4Diethynylbicyclo[2.2.2] octane (BCO) core (Figure 1a). ${ }^{7-9}$ This outcome has been the primary incentive towards the design of crystalline constructs that generate an empty volume that is greater than the volume of revolution of the rotor, the

a. Departament de Química Física i Inorgànica, Universitat Rovira i Virgili, Marcel.Ií Domingo 1, 43007 Tarragona, Spain.Email: antonio.rodriguezf@urv

${ }^{b}$. Institut de Ciència de Materials de Barcelona (ICMAB-CSIC), Campus de la UAB, 08193 Bellaterra, Spain. Email: canadell@icmab.es

c. Laboratoire de Physique des Solides, CNRS UMR 6502, Université de Paris-Sud, Orsay, France.Email: pawel.wzietek@u-psud.fr

d. Laboratoire MOLTECH-Anjou, CNRS UMR 6200, Université d'Angers, 49045

Angers, France.Email: patrick.batail@univ-angers.fr

e. Institute of Solid State Physics, Russian Academy of Sciences, Chernogolovka MD, Russia

† Electronic Supplementary Information (ESI) available: Synthesis, crystal structure, VT ${ }^{1} \mathrm{H}$ spin-lattice relaxation time $\left(T_{1}\right)$, computational details and additional results. Supporting Figs S1-S6. Two movies for selected fragments of the trajectories. Full experimental details for $\mathrm{X}$-ray structure determination and characterisation, CCDC 1893363. See DOI: $10.1039 / x 0 x x 00000 x$ ultimate model system being metal-organic frameworks (MOFs). ${ }^{7,8}$ There, nearly free rotors exist in high dimensions (isotropic), loose surroundings. Meanwhile, case studies combining ${ }^{1} \mathrm{H}$ spin-lattice relaxation ${ }^{9-12}$ with DFT calculations of rotor-rotor and rotor-environment interaction energies are emerging that feature ultra-fast rotors whose rotational barriers may be even lower than $1 \mathrm{kcal} \mathrm{mol}^{-1}$, despite performing in crowded environments. ${ }^{7,9-12}$ Such fluidity of motion in densified surroundings requires soft intermolecular interactions to give way when the temperature is lowered, resulting in lattice instabilities leading to phase transitions. ${ }^{10,11}$ Note that interactions involving polarized halogen bonds are especially prone to undergo such phase transitions. Thus, lattice instabilities are significant in as much as they serve their primary purpose well, which is to avoid adverse (short) intermolecular interactions along the rotational profile, namely $\mathrm{C}-\mathrm{H}^{\cdots}{ }^{\circ} \mathrm{H}-\mathrm{C}$ intermolecular distances much shorter than $2.4 \AA$, that would otherwise impede the motion. ${ }^{10-12}$

We address in this work the case of $\left\{\mathrm{Li}_{4}^{+}\left(\mathbf{1}^{-}\right)_{4}\left(\mathrm{H}_{2} \mathrm{O}\right)_{8}\right\} \bullet 2 \mathrm{DMF}$ (Figures 1 and $\mathrm{S} 1$ ) where four independent rotors are confined into layers instead of the free volume of a MOF. The remarkable finding of this work is that DFT molecular dynamics simulations coupled to variable-temperature (30-300 K) proton spin-lattice relaxation time experiments deliver the same high value for the rotational barriers of all four rotors. Furthermore, we were able to reveal a microscopic model of the mechanism of interrelated rotational motions whereby disrotatory and conrotatory motions in pairs as well as rotational steps of 
single rotators occur within a set of four rotors confined in two-dimensions, one whose complexity could not have been apprehended otherwise.

From a design viewpoint, the present research took off by grasping opportunities for ligand-directed control ${ }^{13-15}$ over rotor environment in framework solids in the wake of the successful desymmetrization of chiral 1,4Diethynylbicyclo[2.2.2] octane rotor (BCO) $)^{16-18}$ (Figure 1a). This led us to prepare the extended, contorted rod $1^{16}$ (Figure 1a) to be engaged with group la metals. Of precisely defined length, 1 has a curvature and helical twist (Figure 1b) that confers much variability in shape compared to the shorter, rigid and linear isonicotinate linker that directs the construction of the lithium(I) salt with an extended, microporous framework reported by Abrahams, Robson and co-workers (Figure 1a). ${ }^{19}$

\section{Results and discussion}

\section{X-ray structure of $\left[\mathrm{Li}_{4}^{+}\left(\mathbf{1}^{-}\right)_{4}\left(\mathrm{H}_{2} \mathrm{O}\right)_{8}\right] \cdot 2 \mathrm{DMF}$}

The compound formulation (Figure S1) was determined by single crystal $\mathrm{X}$-ray diffraction at $120 \mathrm{~K}$ of a thin, merohedrally twinned colorless plate (Figure S2). DMF molecules are disordered over two positions in the lattice with unbalanced, $0.75 / 0.25$ occupancies. One equilibrium position only is found for each of the four rotors in the asymmetric unit. This differs from most crystalline BCO systems investigated so far where a distribution of two ${ }^{9}$ or more ${ }^{10,11}$ equilibrium positions on the same rotor site reflects soft surroundings favoring fast rotation. Since, in addition, the pyridine end groups do not coordinate, preventing a coordination polymer to develop over the third dimension of an open-framework, ${ }^{19}$ a rather dense lattice should therefore be expected, one that somewhat squeezes the rotors in their environments ${ }^{11}$ resulting in rather large energy barriers.

\section{Dense two-dimensional assemblages instead of 3D open} framework

As exemplified in Figure 1, ligand control over the structure is achieved by interdigitation of charge-compensated motifs, formulated $\left\{\mathrm{Li}_{4}^{+}\left({ }^{-} \mathrm{CO}_{2}-\mathrm{Ph}-\mathrm{BCO}-\mathrm{py}\right)_{4}\left(\mathrm{H}_{2} \mathrm{O}\right)_{8}\right\}$, with a cyclic metalrotor core and outer parallel helical ligands, whose built-in steps (Figure $1 \mathrm{~b}$ ) clicks to deliver a neat self-complementary assemblage into robust $(a, b)$ slabs (Figure $1 c)$.

Four independent rotors labeled $A$ to $D$ assemble in ...-A-B-A... and ...-C-D-C-... sequences along two independent chains that lie side-by-side in $(a, b)$ layers as shown in Figure $2 a$ and $2 \mathrm{~b}$. An inventory of $\mathrm{H}$... H rotor-rotor contacts reveals that $(a, b)$ layers are really an assemblage of those pairs of chains, or double chains, (Figures $2 a$ and $2 b$ ), and the pairs interact weakly through a single $\mathrm{H}$... H rotor-rotor interaction at $2.471 \AA$ (dotted red lines in Figure 2a).

In such tangled environments, BCO units are not freely-moving rotors. Instead, any rotor is defined by how it rubs on its surroundings through $\mathrm{H} \cdots \mathrm{H}$ contacts. ${ }^{9,10,11,21}$ For example, analysis of the contacts inventory (Figure $2 \mathrm{c}$ ) reveals the four different environments of the four independent BCO rotators in the asymmetric unit. Therefore, in order to have some hint on the activation energy associated with the rotational motion within the double chains, rotational barriers for rotors $A, B, C$ and $D$ have to be estimated separately. Since, however, the environments of rotors $A$ and $B$ as well as those of $C$ and $D$ are quite similar, only two different relaxation maxima are likely to be distinguishable when it comes to investigate the rotors dynamics by variable-temperature ${ }^{1} \mathrm{H}$ spin-lattice relaxation time experiments.

(a)



(b)


Figure 1. Two-dimensional framework of the lithium salt of rotor $\mathbf{1},\left\{\mathrm{Li}_{4}^{+}\left(\mathbf{1}^{-}\right.\right.$ )$\left._{4}\left(\mathrm{H}_{2} \mathrm{O}\right)_{8}\right\} \bullet 2(\mathrm{DMF})$ : (a) Desymmetrization of 1,4-Diethynylbicyclo[2.2.2] octane (BCO, blue fragment) produces the $1.8 \mathrm{~nm}$, contorted rod 1 ; (b) Chargecompensated, ca. $4 \mathrm{~nm}$ long motif with a cyclic metal-rotor core, $\left\{\mathrm{Li}_{4}^{+}\left(\mathbf{1}^{-}\right)_{4}\left(\mathrm{H}_{2} \mathrm{O}\right)_{8}\right\}$ (see also Figure S1). Note that the two contorted pairs of helical rods $\mathbf{1}$ together with the built-in step confers a self-complementary shape for neat self-assembly into $(a, b)$ slabs; (c) Layer-by-layer packing of slabs along $c^{*}$, normal to the thin colorless plates shown in Figure S1.

\section{Estimating rotational barriers with discrete DFT calculations}

In previous works, ${ }^{10-12,20-22}$ we have shown that the rotational barriers associated with a wide spectrum of crystalline rotors can be reasonably estimated by means of partial geometry optimizations, provided that the positions of all atoms of the rotor itself, as well as those of their near environment, are optimized. The calculated barriers are usually comparable with those emerging from ${ }^{1} \mathrm{H}$ spin-lattice relaxation experiments. We have estimated the rotational barriers for the motion of the rotors highlighted in Figure $2 c$ using the same approach. 

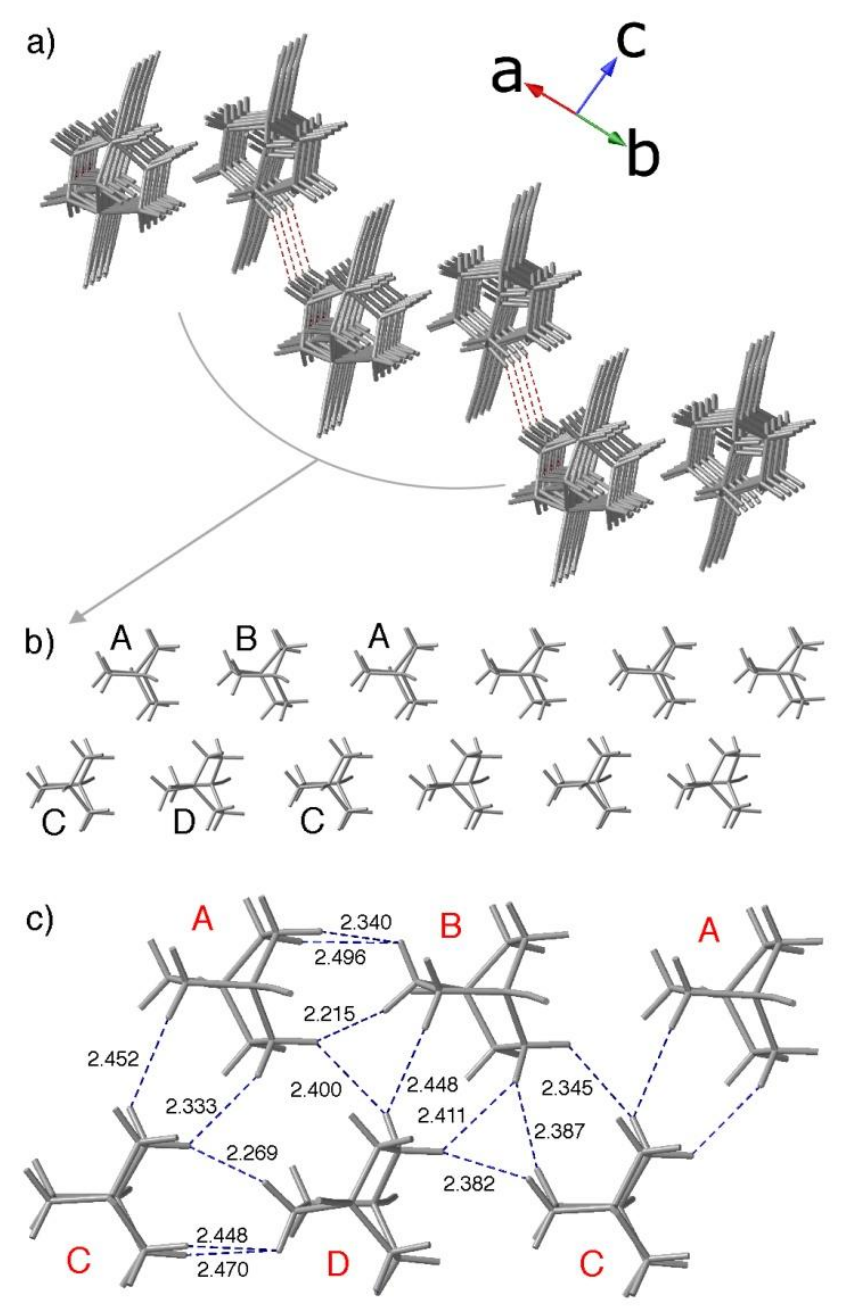

Figure 2. a) The two independent parallel chains of rotors in $(a, b)$ layers are gathered in weakly interacting pairs on account of a large separation of $2.471 \AA$ (dotted red lines); b) $-\mathrm{A}-\mathrm{B}-\mathrm{A}-\ldots$ and ...-C-D-C-... sequences along the two independent chains; c) $\mathrm{H} . . . \mathrm{H}$ contacts shorter than $2.5 \AA$ in the double-chains. Pyridine and benzoate groups are not depicted.

We considered a central rotor surrounded by all neighboring rotors making $\mathrm{H} \cdots \mathrm{H}$ contacts shorter than $2.5 \AA$ in the crystal structure. Since the $\mathrm{H}$ atoms of the pyridine and benzoate groups are not engaged in $\mathrm{H} \cdots \mathrm{H}$ interactions with the rotor blades, they were substituted by planar $\mathrm{CH}_{2}$ groups. In the following we will refer to these reduced rotors as small rotors. For every value of the $\theta$ rotational angle (measured as the HCCC dihedral angle, see Figure 4 a for instance) the $-\mathrm{C}_{8} \mathrm{H}_{12}$ units of the central small rotor as well as those of the neighboring ones were fully optimized. The calculations were carried out for small rotors $A$ and $C$ which according to their environments should lead to activation energies similar to those of $B$ and $D$, respectively. We have employed the same computational details as in previous works. ${ }^{10,21}$ For the two cases the barriers are estimated to be substantially larger, i.e. around $9-10 \mathrm{kcal} \mathrm{mol}^{-1}$ (see Figure $3 \mathrm{a}$ ), than those for similar systems. ${ }^{10-12,20-22}$ a)



b)

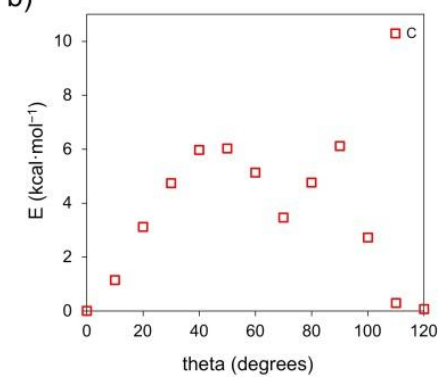

Figure 3. (a) Computed energy profiles for the rotation of small rotors (see text) $A$ and $C$ surrounded by their neighbors where the $-\mathrm{C}_{8} \mathrm{H}_{12}$ - units of the central rotor and those of the neighboring ones were optimized. (b) Computed energy profile for the rotation of small rotor $C$ surrounded by their neighbors when for each value of the rotational angle the structure of the small rotor $C$ and its neighbors were fully optimized.

In both cases $\mathrm{H} \cdots \mathrm{H}$ contacts for the highest point along the energy profile are as short as $2.08 \AA$ when one blade is right in front of another. Note that the two calculated barriers are very similar despite being associated with different environments, a credible indication that a single mechanism applies for the rotational motion of all four rotors.

In contrast with our previous work, ${ }^{9-12,20-22}$ the present rotational barriers seem to be too high. There are several factors which could be at the origin of this fact. For instance, it could be that the rod's curvature changes significantly during the rotational motion thereby affecting the barrier, an aspect not accounted for in the calculations. Since the triple bonds were capped with planar $\mathrm{CH}_{2}$ groups the large aromatic substituents can make an additional contribution to such bending. More importantly, because each rotor rubs strongly on its neighbors by quite short inter-rotor $\mathrm{H} \cdots \mathrm{H}$ distances, any rotor is strongly coupled to the three others. In such case, estimating barriers from independent calculations may prove to be less accurate than in other cases. DFT molecular dynamics simulations have thus been carried out to obtain more reasonable values for the barrier(s) and to provide some hints of a microscopic description of the mechanism of rotation ${ }^{23}$ of the four independent rotors.

\section{Car-Parrinello molecular dynamics suggest lower rotational barriers and significant rotational interrelationship}

Car-Parrinello molecular dynamics (CPMD) ${ }^{24}$ simulations were performed for a periodic layer built out of a monoclinic unit cell formed by four small rotors as in the partial geometry optimizations (see Figure 4a). During a trajectory of 300 ps, no rotation of any of the rotors was observed. This result confirms that the barrier is not smaller than 2-3 $\mathrm{kcal} \mathrm{mol}^{-1}$, as we previously found for crystalline rotors based on similar extended rods, since for such small barriers we should observe some of the rotation events at the time scale of our simulation. Consequently, in order to be able to carry out meaningful molecular dynamics we must turn to some technique allowing an acceleration of the exploration of the energy landscape of the system. We have chosen the metadynamics approach ${ }^{24,25}$ because it offers the choice to 
deliberately activate one or several variables out of a set of collective variables; for example, the rotational motion of one or several rotors. Let us simply remind that during a metadynamics simulation, where it is assumed that the system is appropriately described by the right choice of collective variables, sampling the energy landscape is accelerated via the introduction of an additional bias potential acting on the selected collective variables, thereby discouraging the system to come back to already explored regions. The repeated introduction of the bias potential accelerates the sampling while allowing for a precise estimation of the free energy of the process. $^{25}$



Figure 4. (a) Unit cell with four small rotors used in the Car-Parrinello MD simulations. The atoms of the $\mathrm{H}-\mathrm{C}-\mathrm{C}-\mathrm{C}$ dihedral angle of small rotor $\mathrm{B}$ used as collective variable in the metadynamics run are also represented. (b) Variation of the dihedral angles (theta) of the four rotors during the short metadynamics run. The red line corresponds to the activated dihedral angle (small rotor B) and thus the blue line corresponds to a non-activated small rotor (A). See the movie (small_model_shortMD_39_47ps.mp4) corresponding to the inset in (b) in the Supplementary Information.

We have activated the system, performing first a "short", 51 ps metadynamics run in which we have considered a single collective variable, one $\mathrm{H}-\mathrm{C}-\mathrm{C}-\mathrm{C}$ dihedral angle (see Figure 4a) that describes the rotation of the blades with respect to the axis of one of the small rotors (B) in the unit cell. The estimated free energy barrier for the rotation from one minimum to another is around $6 \mathrm{kcal} \mathrm{mol}^{-1}$, a value which as expected is smaller than the energy estimation obtained from the partial optimizations, which are always an upper bound since the rotors and the blades have some flexibility within the crystal lattice that is not considered in these partial optimizations.

Before trying to gain some chemical insight from this simulation we must recall that for small rotor B the rotational potential has been effectively flattened (i.e. activated) so that although this fact does not influence the rotational barrier estimation, one must be careful when analyzing the trajectories, which should not be treated as those of the unbiased molecular dynamics approaches, which have a welldefined physical significance. ${ }^{26}$ Yet, with this reservation in mind, useful chemical information can be obtained from these trajectories if they are simply treated as a way to explore the kind of rotational events that may occur and thus, to have some plausible microscopical view of the situation in the real system. With this spirit in mind, looking at the results of Figure $4 \mathrm{~b}$ provides some valuable hints concerning rotational motion in crowded media. Shown in this figure is the evolution of the dihedral angle of the four small rotors along the short metadynamics run. The feature to note at this point is that the rotation of the blades of the activated small rotor B (red line in Figure $4 \mathrm{~b}$ ) enforces the rotation of one of the neighboring and non-activated rotors (small rotor $A$, blue line), suggesting that the rotation of the blades of the rotors can be interrelated.

As seen in Figure $4 \mathrm{~b}$, after an apparently individual rotation of the activated small rotor $B$ at around $20-22$ ps there is a complex motion occurring along a long time interval of 8 ps (see inset in Figure 4b) whose final outcome is a conrotatory rotation of activated small rotor $B$ and non-activated small rotor A. Since the motion occurs in quite crowded surroundings and seems to be interrelated, a final disrotatory motion could be expected to be a more "natural" outcome as far as only the A-B pair is implicated in the rotation. However, after examination of the inset of Figure $4 b$ as well as the associated movie (see movie 1 in Supplementary information) it is quite clear that the non-activated small rotors $C$ and $D$ also play an active role in the whole process. These rotors undergo relatively large rotations of around 50-60 degrees and because of the crowded environment interfere with the rotational motion of small rotors $A$ and $B$ leading to the "unexpected" conrotatory output.

Such final conrotatory rotation is thus the result of a complex series of motions implicating the interaction between at least three rotors, something which is favored because the two chains are shifted by one-half repeat unit. As a matter of fact, the final conrotatory motion could also occur as the result of a more "ordered" process where two disrotatory motions implicating a common rotor interfere, as we have found for non-activated rotors in some of our longer simulations to be discussed below. For the time being our conclusion is that the rotation of the blades of different rotors in the present solid 
may be strongly interrelated and the rotational barriers must be lower than obtained in the discrete DFT calculations.

Three relaxation processes revealed by variable-temperature (20$300 \mathrm{~K})$ proton spin-lattice relaxation time experiments, ${ }^{1} \mathrm{H} T_{1}^{-1}$

Variable-temperature proton spin-lattice relaxation experiments performed on static crystalline samples in a wide temperature range have recently demonstrated great promise to gain insight into the dynamics of crystalline rotors and, when appropriate, help disentangle multiple relaxation processes occurring at the same spin temperature..$^{7-12}$ As typically found in molecular solid-state NMR, strong dipolar $\mathrm{H}$ $\mathrm{H}$ interactions are responsible for broad, featureless proton spectra. Dipolar coupling however generates fast spin diffusion ensuring that all spins quickly reach equilibrium at the same temperature. This is important because then the relaxation of large collections of ${ }^{1} \mathrm{H}$ spins is well described by a single exponential where the relaxation rate $T_{1}^{-1}$ measured at a given temperature is simply a weighted average of the contributions of all processes associated with all individual moving units. Given that relaxation processes that come from similar moving parts can be modeled the same way - for example, each BCO rotor is modeled with the same standard Kubo-Tomita (KT) expression (Eq. 1-2) - the weights are simply proportional to the respective numbers of contributing protons and are therefore readily available from the chemical formulation and crystal structure (see Figure S1c). Although we have to resort to modeling the whole $T_{1}$ curve vs temperature in order to deconvolute various contributions, the information contained in the relative weights makes such global fit quite robust. ${ }^{9}$

Here, variable-temperature $(20-300 \mathrm{~K}){ }^{1} \mathrm{H} \mathrm{T}_{1}^{-1}$ data (Figure 5) are dominated by a broad relaxation whose maxima would appear to occur above room temperature, alluding to a large energy barrier for this process. In addition, two maxima with much smaller yet different intensities are identified at low temperature, around $55 \mathrm{~K}$ and $125 \mathrm{~K}$. This pair of peaks is recognized as a clear example of quantum-tunneling relaxation of a methyl group in the solid state described by Allen and Clough. ${ }^{27}$ In Figure 5 this contribution to the fit (solid line) has been obtained applying the model developed by these authors (Table S1). ${ }^{27}$ The best fit was obtained assuming a slight distribution of each of these two energy barriers (here a Gaussian distribution with a relative width of $16 \%$ ) reflecting the disorder affecting the DMF molecules in the lattice (Figure 5).

The high temperature process, assigned altogether to the four $\mathrm{BCO}$ rotors, will be fitted with one standard Kubo-Tomita expression:

$$
T_{1}^{-1}=C\left[\tau_{\mathrm{c}}\left(1+\omega_{0}{ }^{2} \tau_{\mathrm{c}}^{2}\right)^{-1}+4 \tau_{\mathrm{c}}\left(1+4 \omega_{0}^{2} \tau_{\mathrm{c}}^{2}\right)^{-1}\right],
$$

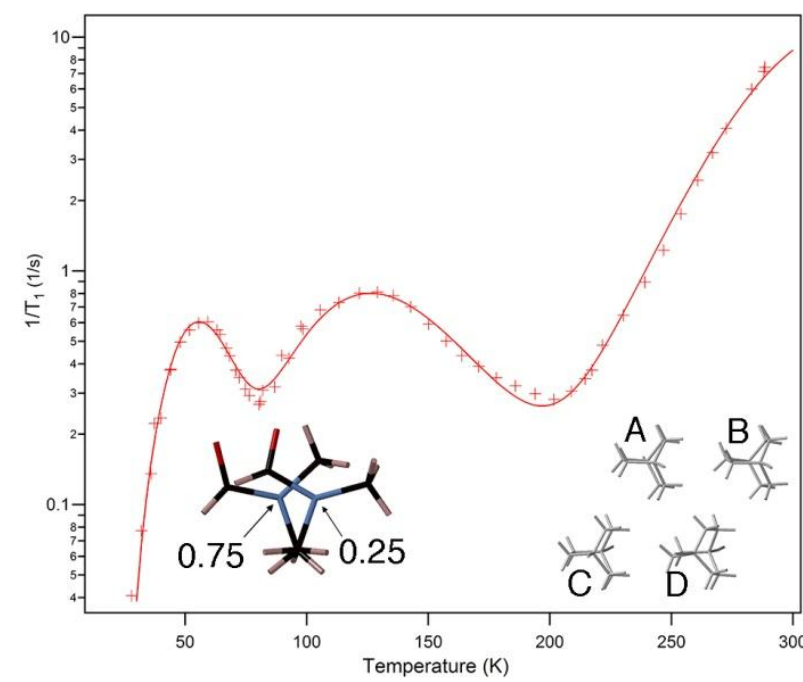

Figure 5. Variable-temperature ${ }^{1} \mathrm{H}$ spin-lattice relaxation time $\mathrm{T}_{1}{ }^{-1}$ at $57 \mathrm{MHz}$ carried out on a static polycrystalline sample of $\left[\mathrm{Li}^{+}{ }_{4}\left(\mathbf{1}^{-}\right)_{4}\left(\mathrm{H}_{2} \mathrm{O}\right)_{8}\right] \bullet 2 \mathrm{DMF}$. The red line represents the fit of the data to the Kubo-Tomita expression relevant for the $A B C D$ quartet, and to Allen and Clough equation ${ }^{20}$ describing the quantumtunneling relaxation of the two methyl groups of DMF molecules (see text and Table S1).

Where $\omega_{0}$ is the proton NMR frequency and $\tau_{c}$ is the correlation time described by the Arrhenius equation (Eq. 2) in terms of characteristic activation energies $\left(E_{\mathrm{a}}\right)$ and preexponential factors $\left(\tau_{0}^{-1}\right)$ :

$$
\tau_{\mathrm{c}}^{-1}=\tau_{0}^{-1} \exp \left(-E_{a} / R T\right)
$$

As the constant $C$ in (Eq. 1) only depends on the moving part, it is the same for each BCO rotor site. Therefore, keeping in mind that the measured relaxation is averaged by spin diffusion, the intensity of each $\mathrm{BCO}$ contribution merely scales with the ratio of net number of moving ${ }^{1} \mathrm{H}$ on each independent $\mathrm{BCO}$ site relative to the total number of protons (see Figure S1c). ${ }^{28}$ In the present case, since our measurements do not reach the high temperature maximum, this scaling serves us well to estimate the expected intensity of $T_{1}^{-1}$ in order to allow for an unambiguous fit of the activation energy.

With four different rotors environments (see Figure $2 \mathrm{c}$ ), as many rotational barriers are expected to contribute to the high temperature relaxation processes. However, with incomplete data at high temperature it is not possible to resolve these individual processes and the analysis has been limited to a fit with a single activation energy. Also, for the same reason, we prefer to stiffen the fit using an independent determination of the scaling factor $C$ in Eq. 1 rather than relying on the standard procedure where it is obtained solely from the fit together with $E_{\mathrm{a}}$ and $\tau_{0}$. This factor is readily found with the knowledge that, in addition to the four rotors $A-D$, that involves the 48 moving protons of the three $\mathrm{CH}_{2}-\mathrm{CH}_{2}$ blades, there are a total of 62 additional protons from pyridine (32), water (16) and DMF (14) molecules not involved in the relaxation at room temperature, the contribution of immobile protons being counted as zero in the total relaxation rate. ${ }^{28}$ 
a)

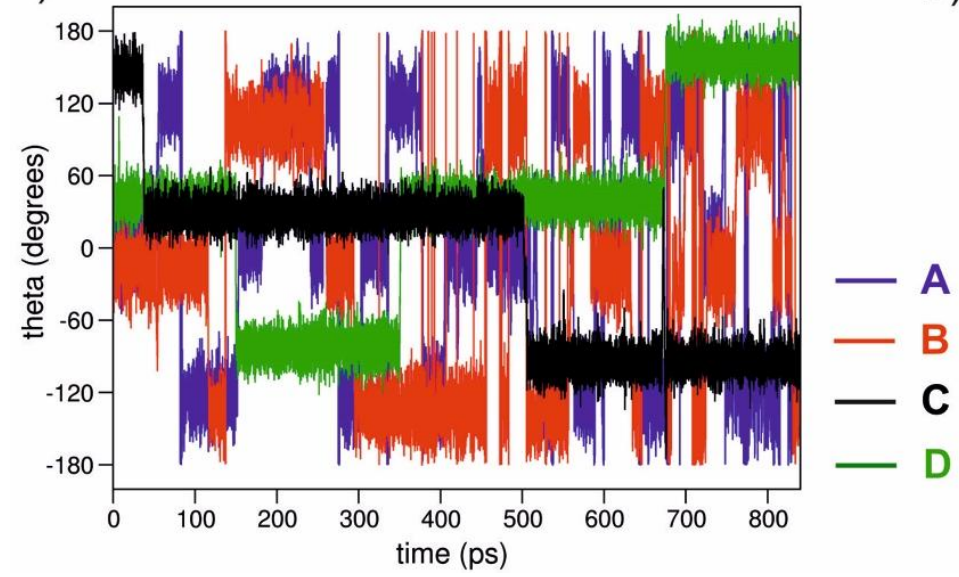

b)

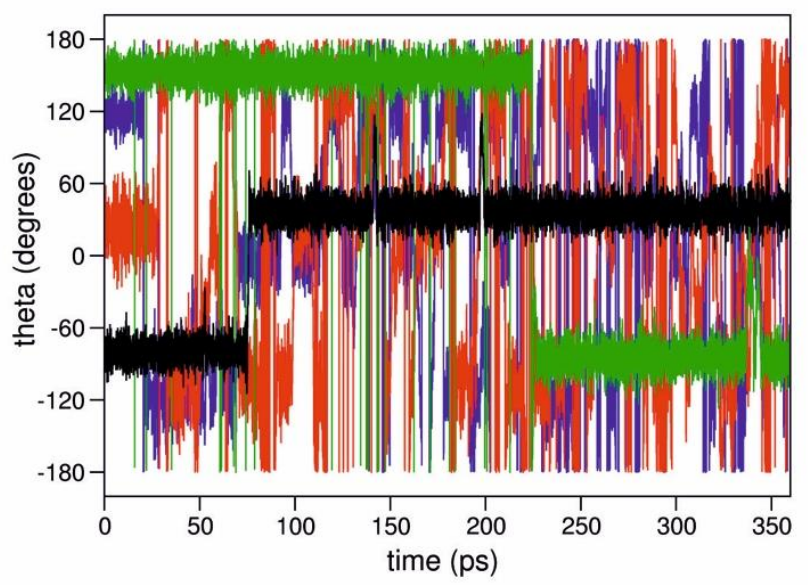

Figure 6. Variation of the four dihedral angles during the metadynamics trajectory of the layer with unit cell of (a) four small rotors and (b) four large rotors, where the dihedral angles of both rotors $A$ (blue) and B (red) are activated. The dihedral angles of rotors C (black) and D (green) are not activated.

Note that (Figure 5) the methyl groups contribution becomes negligible at room temperature, therefore at high temperature we may consider that the only source of relaxation come from the BCO units. The maximum of $T_{1}^{-1}$ could then be readily calculated with the high confidence provided by a control fit of earlier data based on a similar proton inventory for two other BCO systems. ${ }^{10,28}$ These latter data sets gave the same result within $5 \%$, thereby validating this procedure. The fit shown in Figure 5 is obtained with an energy barrier of $3000 \mathrm{~K} / 6 \mathrm{kcal}$ $\mathrm{mol}^{-1}$ for the broad high temperature relaxation processes.

Taken together, the design, structure and variabletemperature solid-state NMR dynamic deliver a neat yet too broad a picture as it does not allow to resolve the contribution of the individual relaxation rates of each of the four rotors, in contrast with recent examples where several maxima were discernible and deconvoluted. ${ }^{10,11}$ Here, the actual dynamics of the four intertwined BCO units are hidden, buried deep and seemingly inaccessible inside a broad, featureless relaxation maximum. We now come back to Car-Parrinello molecular dynamics to have a theoretical estimation of the rotational barriers.

\section{A single rotational barrier $\left(6 \mathrm{kcal} \mathrm{mol}^{-1}\right)$ is also obtained from Car- Parrinello molecular dynamics}

We have performed a long metadynamics run considering two dihedral angles for two different small rotors ( $A$ and $B$, see Figures $2 b$ and $4 a$ ) as collective variables. These two activated dihedral angles (blue line, small rotor $A$; red line, small rotor $B$ in Figure 6a) change significantly during the long 840 ps trajectory. Interestingly, the non-activated dihedrals do also change (black line, small rotor $C$ and green line, small rotor $D$, in Figure 6a), at a lower frequency though, simply because they are non-activated.

We have added all the Gaussians (bias potentials) introduced during the metadynamics in order to estimate the free energy surface (see Figure 7a) with respect to the two considered collective variables (the two dihedral angles). We observe the wells of the periodic equivalent minima, separated by 120 degrees, for the two dihedral angles. The estimation for the barrier that connects two equivalent minima is around $6 \mathrm{kcal}$ $\mathrm{mol}^{-1}$, very similar to the prediction made with the metadynamics with a single collective variable. Note that the energy barrier separating two minima is essentially the same when moving along the horizontal/vertical direction (individual rotation) or when moving along the diagonal (gearing rotation of two rotors). We thus conclude that gearing, non-gearing and more complex combined rotations can occur although in such very dense surroundings they proceed with similar energy barriers.

In Figure S3, the change of position of a single $\mathrm{C}$ atom for each of the rotors is represented as the 820 ps simulation for the small rotors proceeds in order to visualize the trajectories of these four $\mathrm{C}$ atoms. For small rotors whose rotations have not been activated ( $C$ and $D$, black and green positions in Figure S3), rotations of around 120 degrees are essentially observed. The activated small rotors ( $A$ and $B$, blue and red), on the other hand, move much more freely, as it must be because they have been activated, and of course span a more continuous set of positions. In particular, we can see as if there were two sets of positions of the blades separated by 60 degrees (see scheme in Figure S5b), a feature reminiscent of that found in $\mathrm{X}$-ray structures of rotors with multiple equilibrium positions in analogous systems. ${ }^{10}$ What Figures $6 a$ and S3 (as well as Figures $6 \mathrm{~b}$ and $\mathrm{S} 5$ for the large rotors trajectory, see below) make clear is that both activated and non-activated rotors have gone through a large number of rotations so that the sampling of the energy landscape of the periodic layer is very extensive and thus the free energy landscape is quite accurate. A question to consider is whether capping the rod with aromatic stoppers (pyridine and benzoate) affects the barrier. 
a)

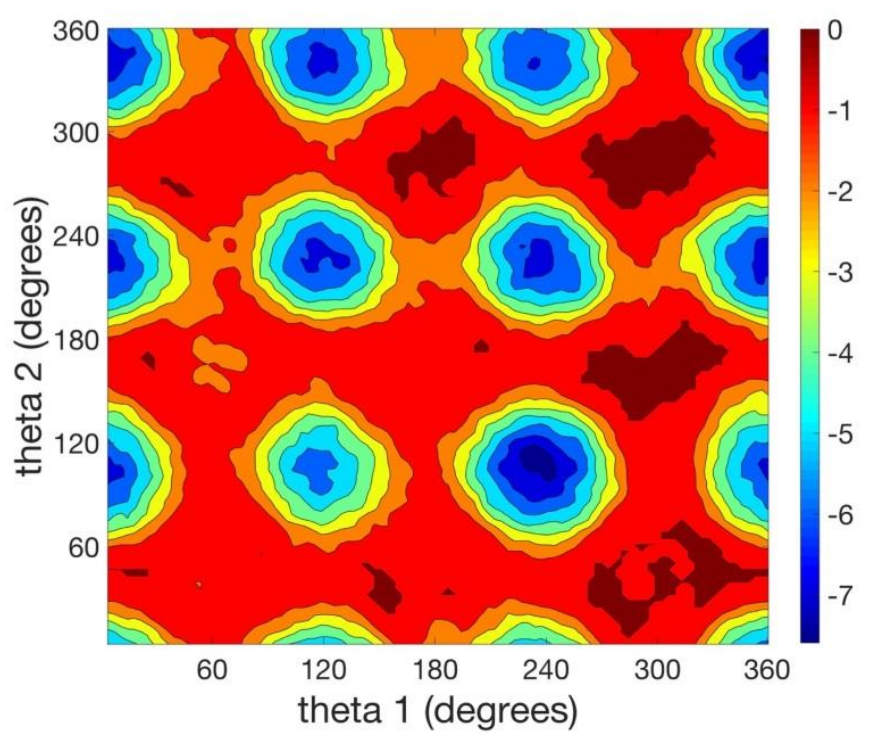

b)



Figure 7. Free energy surface estimated from the metadynamics run of the unit cell with (a) four small rotors (Figure 4a) and (b) four large rotors (Figure S4). The units in the color bar are $\mathrm{kcal} \mathrm{mol}^{-1}$. In (b) the principal equivalent minima (m1) are separated by 120 degrees in each of the two coordinates, theta 1 and theta 2 . In the middle of four principal $\mathrm{m} 1$ minima, new incipient minima $(\mathrm{m} 2)$ appear at the time scale of our simulation. These $\mathrm{m} 2 \mathrm{minima}$ are 120 degrees apart and separated from $m 1$ by $\Delta$ theta $1=\Delta$ theta $2=60$ degrees.

An analogous study with a more realistic system (refereed to from now on as large rotors) was performed to that effect with the benzoate and the pyridine end-groups of the real molecules modeled each by a phenyl group (see Figure S4). These large rotors show a larger flexibility than the former, smaller ones, allowing the rotor axis to distort/bend to a higher degree so that the blades would end up further apart (with larger $\mathrm{H} \cdots \mathrm{H}$ contacts). Let us remind that in the crystal these substituents do not directly interact with the rotor blades. A long metadynamics run (360 ps) was performed using the same two dihedral angles as collective variables. The results, shown in Figures $6 b$ and $7 b$, are essentially similar to those found for the smaller system. The activated dihedral angles (Figure 6b) change significantly during the trajectory, whereas the non-activated ones do not stay still but also change in concert with the activated ones. The free energy surface calculated as a function of the two dihedral angles (Figure $7 \mathrm{~b}$ ) also shows equivalent minima (m1) separated by 120 degrees. The barriers that connect two equivalent minima are also estimated to be around $6 \mathrm{kcal} \mathrm{mol}^{-1}$, so the effect of the aromatic stoppers seems not to be crucial for predicting the barrier. Note however that now the diagonal path implicates a somewhat higher energy barrier. It is interesting to point out that in the middle of each square of four equivalent minima, another incipient minima appear, which would correspond to the second set of positions (m2) separated by 60 degrees from the principal minima observed in Figure S5. A larger simulation would lead to better estimation of the relative free energies between these two minima and of the barrier along the diagonal path corresponding to the gearing dynamics. We thus conclude that the motion of the rotors in $\left\{\mathrm{Li}_{4}^{+}\left(\mathbf{1}^{-}\right)_{4}\left(\mathrm{H}_{2} \mathrm{O}\right)_{8}\right\} \cdot 2 \mathrm{DMF}$ involves different rotations of pairs of rotors as well as individual rotations coupled in a complex way, with a sizeable rotational barrier around $6 \mathrm{kcal} \mathrm{mol}^{-1}$.

To gain some microscopic hints about rotation in crowded media we have examined the runs of Figures $6 \mathrm{a}$ and $6 \mathrm{~b}$ in the spirit outlined above concerning metadynamics trajectories. We have found that three different types of rotational motions, which involve activated and/or non-activated rotors, actually occur: (i) rotation of a pair of rotors with a final disrotatory output ("disrotatory"), (ii) rotation of a pair of rotors with a final conrotatory output ("conrotatory"), and (iii) the rotation of a single rotor ("individual"). Note that we use the terms "conrotatory" and "disrotatory" to qualify the final outcome of a motion leading to the rotation of two adjacent rotors with the same or different signs. The two rotors may be of the same chain or from different chains. Any of the two types, "disrotatory" and "conrotatory", embrace a large series of different and complex rotational motions leading to the same final output. Note also that we use the "individual" term to indicate that the large rotational motion of a given rotor is not associated with the large rotational motion of an adjacent rotor although all adjacent rotors influence it. Shown in Figures S6 and S7 are enlarged views of some portions of the metadynamics trajectories where we have highlighted some typical examples of all of them. It is important to note that among the disrotatory motions sampled in the two trajectories some of them can be seen as disrotatory gearing processes 
even in such crowded medium (see for instance the highlighted region between 505 and 506 ps in Figure S6a).

Note that in our periodic calculation, 1D gearing involving two rotors of the same ...-A-B-A-.. chain, which we have observed for instance at several points of the trajectories in Figure 6, may suggest that a concerted, extended gearing along the whole chain can occur. This is not so however, because as clearly shown in Figure $2 c$ the different A-B pairs are not related through short $\mathrm{H}$...H contacts and thus from the viewpoint of their rotational motion the ...-A-B-A-... chain is simply a series of quasi-isolated A-B pairs. This would be clear in metadynamics runs using a larger number of unit cells although it would practically not affect the rotational barrier estimation.

Finally, let us note that even if the metadynamics runs have been carried out using periodic boundary conditions along the two directions of the layer, the fact that there are no $\mathrm{H}$...H contacts in the crystal structure implicating rotational units shorter than $2.4 \AA$ suggests that the rotational motion in one double chain does not interfere with those in the adjacent ones as confirmed by the fact that no short $\mathrm{H}$... H contacts of this type are created along the trajectory. In other words, double chains behave independently and this is one of the appealing features of the present system as it implies that by appropriately tuning interactions (through variations of the terminal substituents or the interlayer units), it should be possible to manipulate the inter-chain coupling and build a series of layered systems ranging from those where chains behave independently, to layered systems of double chains, triple chains, etc.

\section{Discrete DFT calculations can also provide a good estimation of the rotational barriers}

A striking result comes out of the DFT metadynamics study: the calculated rotational barrier always amounts to about 6 $\mathrm{kcal} \mathrm{mol}^{-1}$ regardless of the way the donor layers have been modelled or the time scale of the simulation run. This finding supports the use of a single activation energy to analyze the proton spin-lattice relaxation time experiments. In addition, the calculated rotational barrier is in excellent agreement with the value obtained from these experiments. Capping the rod with pyridine and benzoate substituents clearly does not control the height of the barrier. Note that the rod curvature imposes the two triple bonds to bend, following the rotational motion during all metadynamics runs. This immediately suggests that discrete DFT calculations of Figure 3a are marred by the neglect of this additional degree of freedom which in very crowded environments apparently plays a substantial role in lowering the rotational barrier.

Thus, we have repeated the discrete DFT calculations of Figure 3a by fully optimizing the structure of the central rotor and all its neighbors. As shown in Figure $3 b$, the calculated energy barrier is now $6 \mathrm{kcal} \mathrm{mol}^{-1}$ in full agreement with both the molecular dynamics study and the spin-lattice relaxation experiments. Thus, discrete DFT calculations although less informative (but less computationally expensive) than DFT metadynamics studies can still provide good estimations of the rotational barriers in crowded situations providing that the appropriate degrees of freedom are considered.

\section{Conclusions}

We have designed the metal-organic rotor, $\left\{\mathrm{Li}_{4}^{+}\left(\mathbf{1}^{-}\right.\right.$ )$\left._{4}\left(\mathrm{H}_{2} \mathrm{O}\right)_{8}\right\} \bullet 2 \mathrm{DMF}$, and reported on how Car-Parrinello molecular dynamics simulations and variable-temperature ${ }^{1} \mathrm{H}$ spin-lattice experiments nicely complement each other to unravel a complex dynamics within the dense layered surroundings of four independent crystalline rotors. Underneath a broad high temperature relaxation process a strongly interrelated series of rotational motions occurs leading to disrotatory and conrotatory rotations in pairs of rotors, and rotational motion of individual rotors all occurring with similar, sizeable rotational barriers of $6 \mathrm{kcal} \mathrm{mol}^{-1}$. Even in the crowded rotational environment of the present salt geared disrotatory motions are found. Looking forward, we underline the appealing ability to chemically manipulate the transverse coupling to sequentially increase the number of interacting chains of rotors in a slab which would advance further the knowledge of rotational motion at the nanoscale in dense media. Another attractive target would be to slightly soften the rotor-environment interactions to see if zones of extended gearing can be induced. From a broader perspective, combining complementary relaxation time experiments and simulations, as exemplified here, may vastly increase the diversity and tunability of available crystalline rotors, allowing for greater control over the dynamic properties of nanomachines.

\section{Conflicts of interest}

There are no conflicts to declare.

\section{Acknowledgements}

We are indebted with Dr. Jordi Faraudo (ICMAB) for an illuminating discussion. Work in Bellaterra and Tarragona was supported by the Spanish MICIU (Projects PGC2018-096955-BC44 and CTQ2017-87269-P) and Generalitat de Catalunya (2017SGR1506 and 2017SGR629). E.C. acknowledges support of the Spanish MINECO through the Severo Ochoa Centers of Excellence Program under Grant SEV-2015-0496. This research was funded by the CNRS, the University of Angers, the University of Paris-Sud, Orsay; the Région des Pays de la Loire Grant MOVAMOL. C. L. thanks the CNRS and the Région des Pays de la Loire for a Ph. D. grant and post-doctoral Fellowship. Work in Chernogolovka was carried out within the state task of ISSP RAS with partial support from the joint CNRS-Russian Federation grants PICS 6028 and RFBR-CNRS 12-03-91059. 


\section{Notes and references}

1 A. C. Balazs, P. Fischer, A. Sen, Acc. Chem. Res. 2018, 51, 2979-2979.

2 I. Ortiz-Rivera, M. Mathesh, D. A. Wilson, Acc. Chem. Res. 2018, 51, 1891-1900.

3 J. A. Abendroth; O. S. Bushuyev, P. S. Weiss, C. J. Barrett, ACS Nano 2015, 9, 7746-7768.

4 S. Erbas-Cakmak, D. A. Leigh, C. T. McTernan, A. L. Nussbaumer, Chem. Rev. 2015, 115, 10081-10206.

5 S. R. Domingos, A. Cnossen, W. J. Buma, W. R. Browne, B. L. Feringa, M. Schnell, Angew. Chem. Int. Ed. 2017, 56, 1120911212.

6 L. Catalano, P. Naumov, CrystEngComm 2018, 20, 5872-5883.

7 C. S. Vogelsberg, F. J. Uribe-Romo, A. S. Lipton, Y. Song, K. N. Houk, S. Brown, M. A. Garcia-Garibay, PNAS, 2017, 114, 1361313618.

8 A. Comotti, S. Bracco, P. Sozzani, Acc. Chem. Res. 2016, 49, 1701-1710.

9 C. Lemouchi, C. S. Vogelsberg, S. Simonov, L. Zorina, P. Batail, P.; S. Brown, M. Garcia-Garibay, J. Am. Chem. Soc. 2011, 133, 6371-6379.

10 S. Simonov, L. Zorina, P. Wzietek, A. Rodríguez-Fortea, E. Canadell, C. Mézière, G. Bastien, C. Lemouchi, M. A. GarciaGaribay, P. Batail, Nano Lett. 2018, 18, 3780-3784.

11 C. Lemouchi, H. M. Yamamoto, R. Kato, R.; S. Simonov, S.; L. Zorina, L.; A. Rodríguez-Fortea, A.; E. Canadell, E.; P. Wzietek, K. Iliopoulos, D. Gindre, M. Chrysos, P. Batail, Cryst. Growth Des. 2014, 14, 3375-3383.

12 C. Lemouchi, C. Mézière, L. Zorina, S. Simonov, A. RodríguezFortea, E. Canadell, P. Wzietek, P. Auban-Senzier, C. Pasquier, T. Giamarchi, M. A. Garcia-Garibay, P. Batail, P., J. Am. Chem. Soc. 2012, 134, 7880-7891.

13 H. H.-M.Yeung, W. Li, P. L. Saines, K. J. Thomas T. K. J. Köster, C. P. Grey, A. K. Cheetham, Angew. Chem. Int. Ed. 2013, 52, 5544 $-5547$.

14 C. Janiak, Dalton Trans. 2003, $2781-2804$.

15 D. Banerjee, J. B. Parise, Cryst. Growth Des., 2011, 11, 4704-

4720.

16 C. Lemouchi, P. Batail, Beilstein J. Org. Chem. 2015, 11, 18811885.

17 G. M. Locke, S. S. R. Bernhard, M. O. Senge, Chem. Eur. J. 2019, 25, 4590-4647.

18 J. Kaleta, G. Bastien, I. Císaová, P. Batail, P.; J. Michl, , Eur. J. Org. Chem. 2018, 5137-5142.

19 B. F. Abrahams, M. J. Grannas, T. A. Hudson, R. Robson, Angew. Chem. Int. Ed. 2010, 49, 1087-1089.

20 C. Lemouchi, I. Iliopoulos, L. Zorina, S. Simonov, P. Wzietek, P.; T. Cauchy, A. Rodríguez-Fortea, E. Canadell, J. Kaleta, J. Michl, D. Gindre, M. Chrysos, P. Batail, P., J. Am. Chem. Soc. 2013, 135, 9366-9376.

21 G. Bastien, C. Lemouchi, M. Allain, P. Wzietek, A. RodríguezFortea, E. Canadell, K. Iliopoulos, D. Gindre, M. Chrysos, P. Batail, CrystEngComm 2014, 16, 1241-1244.

22 J. Kaleta, J. Michl, C. Mézière, S. Simonov, L. Zorina, P. Wzietek, A. Rodríguez-Fortea, E. Canadell, P. Batail, CrystEngComm 2015, 17, 7829-7834.

23 For some interesting molecular dynamics studies along the same line although not using a first-principles DFT approach, see: (a) A. R. Britcher, J. H. Strange, J. Chem. Soc. Faraday Trans. 2, 1978,74, 1767-1777. (b) H. E. Kerr, L. K. Softley, K. Suresh, A. Nangia, P. Hodgkinson, E. I. Radosavljevic CrystEngComm 2015, 17, 6707-6715. (b) A. J. llott, S. Palucha, A. S. Batsanov, M. R. Wilson, P. Hodgkinson, J. Am. Chem. Soc. 2010, 132, 1451795185.

24 R. Car, M. Parrinello, Phys. Rev. Lett. 1985, 55, 2471.
25 (a) A. Laio, M. Parrinello, Proc. Natl. Acad. Sci. USA 2002, 99, 12562-12566; (b) A. Laio, A. Rodriguez-Fortea, L. Gervasio, M. Parrinello, J. Phys. Chem. B 2005, 109, 6714-6721.

26 A. J. llott, S. Palucha, P. Hodgkinson, M. R. Wilson, J. Phys. Chem. B 2013, 117, 12286-12295.

27 P. S. Allen, S. Clough, Phys. Rev. Lett. 1969, 22, 1351-1353.

28 See Equation 3 in the SI for ref. 10. 